\title{
CERTAIN MULTIPLE VALUED HARMONIC FUNCTION
}

\author{
L. A. CAFFARELLI ${ }^{1}$
}

Abstract. The difference equation $v\left(x^{+}\right)-v(x)=u(x)$ is solved for any harmonic $u$ in the covering space of an unknotted curve.

The purpose of this note is to answer a problem posed by $H$. Lewy [2]. The situation is as follows: Given is a curve $\Gamma$ diffeomorphic, in $\mathbf{R}^{3}$, to a circle. That is, we have a diffeomorphism $\varphi: \mathbf{R}^{3} \rightarrow \mathbf{R}^{3}$ that maps $\Gamma$ onto a circle $C$.

If we now consider the universal covering space $S$, of $\mathbf{R}^{3} \sim \Gamma$, it is asked if given a function $u$, harmonic on $S$, we can find a harmonic function $v$, such that $v\left(x^{+}\right)-v(x)=u(x)$ (where $x^{+}$denotes the point obtained from $x$ in $S$ following a path $\delta$ whose projection $\pi(\delta)$ in $\mathbf{R}^{3} \sim \Gamma$ is a closed curve that loops $\Gamma$ once). We are going to prove that such a function $v$ exists.

PROof. The proof is a modification of the proof in [2], to make an integral convergent.

More precisely, let us consider, as in [2], the disk $D$ in $\mathbf{R}^{3}$ that has $C$ as boundary and let $\sigma=\varphi^{-1}(D)$. We will consider neighborhoods $U_{n}$ of $\Gamma$,

$$
U_{n}=\left\{x: d(x, \Gamma) \leqq 2^{-n}\right\} .
$$

By means of the diffeomorphism, for $n_{0}$ large enough we can construct a continuous mapping $\tilde{x}(x): U_{n_{0}} \rightarrow \Gamma$ such that

$$
|\tilde{x}(x)-x| \leqq K d(x, \Gamma) \quad(K \text { a constant }) .
$$

$B_{1}$ will denote a ball verifying $\left(U_{0} \cup \sigma\right) \subset B_{1}$.

Let us consider now the two consecutive leaves $S_{1}, S_{2}$ of $S$ that are between the copies $\sigma_{0}$ and $\sigma_{2}$ of $\sigma$ on $S$ and have the copy $\sigma_{1}$ as common boundary.

Then, in the compact set

$$
W_{n}=\left[\left(S_{1} \cup S_{2}\right) \cap \pi^{-1}\left(B_{1}\right)\right] \sim \pi^{-1} U_{n}
$$

there exists a $\lambda_{n}$ such that

$$
|u| \leqq \lambda_{n}
$$

(As befcre $\pi$ denotes the canonical projection $\pi: S \rightarrow \mathbf{R}^{3} \sim \Gamma$.) Any first derivative of $u$ is therefore bounded in $W_{n}$ by some $\lambda_{n}{ }^{\prime}$. Let

Received by the editors October 31, 1974 and, in revised form, January 12, 1975.

AMS (MOS) subject classifications (1970). Primary 31 B05.

Key words and phrases. Harmonic function, covering space.

1 Fellow of the Consejo Nacional de Investigaciones Cientificas y Tecnicas, Republica de Argentina. 


$$
\bar{\lambda}_{n}=\max \left(\lambda_{n}, \lambda_{n}{ }^{\prime}\right) .
$$

We are going to consider the function

$$
\begin{aligned}
v(x)=\frac{1}{4 \pi} \int_{\sigma \sim U_{n_{0}}}\left(u(y) \frac{\partial}{\partial \nu_{y}} V-V \frac{\partial}{\partial \nu_{y}} u(y)\right) d \sigma(y) \\
+\sum_{k=n_{0}}^{\infty} \int_{\sigma \cap\left(U_{k} \sim U_{k+1}\right)}\left(u(y) \frac{\partial}{\partial \nu_{y}}\left(V-V_{k}\right)(x, y)\right. \\
\left.-\left(V-V_{k}\right)(x, y) \frac{\partial}{\partial \nu_{y}} u(y)\right) d \sigma(y)
\end{aligned}
$$

where $V(x, y)=1 /|x-y|$ and $V_{k}$ is a suitable Taylor's polynomial of $V$ around the point $\tilde{y}$ (as defined in (2)). The values of $u$ are taken over the copy $\sigma_{1}$ of $\sigma$, bounding $S_{1}$ from $S_{2}$. Let us then recall the following developments:

$$
\frac{1}{|x-y|}=\frac{1}{\left|x-y_{0}\right|}\left(\sum_{0}^{\infty} P_{n}(\cos \theta)\left(\frac{\left|y-y_{0}\right|}{\left|x-y_{0}\right|}\right)^{n}\right)
$$

convergent for $\left|y-y_{0}\right|<\left|x-y_{0}\right|, \sup _{|u| \leqq 1}\left|P_{n}(u)\right|=1$ with $\theta$ the angle between $x-y_{0}$ and $y-y_{0}$.

$$
\nabla_{y} \frac{1}{|x-y|}=\frac{1}{\left|x-y_{0}\right|^{3}} \sum_{1}^{\infty}\left(P_{n-1}^{\prime}(u)\left(y-y_{0}\right)-P_{n}^{\prime}(u) \frac{\left|y-y_{0}\right|}{\left|x-y_{0}\right|}\left(x-y_{0}\right)\right)
$$

$$
\cdot\left(\frac{\left|y-y_{0}\right|}{\left|x-y_{0}\right|}\right)^{n-2}
$$

with $u=\cos \theta$ again convergent for $\left|y-y_{0}\right|<\left|x-y_{0}\right|, \sup _{|u| \leqq 1}\left|P_{n}^{\prime}(u)\right|$ $\leqq n(n+1) / 2$.

Each term of (6) and (7) is a harmonic polynomial (see [1, pp. 124 and 142]).

Turning back to (5) we choose $V_{k}$ to be the development (6) with $y_{0}=\tilde{y}(y)$ up to an order $l_{(k)}$ verifying

$$
\bar{\lambda}_{k} 2^{-l_{(k) / 2}}<2^{-k} .
$$

Then if $x \notin U_{m} \cup \sigma_{1}$ and $B(x)$ is a ball centered at $x$ with $\overline{B(x)} \cap\left(U_{m} \cup \sigma_{1}\right)$ $=\varnothing$, for $y \in U_{k}$, with $k \geqq \max \left[K(m+1), n_{0}\right]$, we have, for any $x^{\prime} \in B(x)$,

and

$$
\left(V-V_{k}\right)\left(x^{\prime}, y\right) \leqslant \frac{C 2^{-l}(k) / 2}{\left|x^{\prime}-\tilde{y}\right|} \leqslant C 2^{m} 2^{-l(k)}
$$

where $C$ and $C^{\prime}$ denote constants. Hence, the first $\max \left[K(m+1), n_{0}\right]$ terms of (5) are bounded harmonic functions on $B$, and the remaining terms give us, on $B$, an absolutely and uniformly convergent series of harmonic functions. Now, if $B$ is a small ball intersecting $\sigma$ (with $\bar{B} \cap \Gamma=\varnothing$ ), and we remove from (5) the integral 


$$
\frac{1}{4 \pi} \int_{B \cap \sigma}\left(u(y) \frac{\partial}{\partial \nu_{y}} V(x, y)-V(x, y) \frac{\partial}{\partial \nu_{y}} u(y)\right) d \sigma
$$

(which involves only a finite number of terms of (5)), the remaining terms give us again an absolutely and uniformly convergent series of harmonic functions in any closed subball of $B$. But as in [2], (11) gives us the desired jump in $u(x)$ of the function $v$ across $\sigma$, and that completes the proof.

\section{REFERENCES}

1. O. D. Kellogg, Foundations of potential theory, Dover, New York, 1953.

2. Hans Lewy, Generalization of a theorem on the spatial angle, Russian Math. Surveys 26 (1971), no. 2, 131-137.

Department of Mathematics, University of Minnesota, Minneapolis, Minnesota 55455 